\title{
Partition dependence in consumer choice: Perceptual groupings do not reliably shape decisions
}

\author{
Sheri Reichelson ${ }^{1} \cdot$ Alexandra Zax $^{1} \cdot$ Ilona Bass ${ }^{1,2}$ - Andrea L. Patalano ${ }^{1}$. \\ Hilary C. Barth ${ }^{1}$
}

Published online: 9 June 2017

(C) Psychonomic Society, Inc. 2017

\begin{abstract}
The partitioning of options into arbitrary categories has been shown to influence decisions about allocating choices or resources among those options; this phenomenon is called partition dependence. While we do not call into question the validity of the partition dependence phenomenon in the present work, we do examine the robustness of one of the experimental paradigms reported by Fox, Ratner, and Lieb (Journal of Experimental Psychology: General, 134, 538$551,2005$, Study 4$)$. In three experiments $(N=300)$ conducted here, participants chose from a menu of perceptually partitioned options (varieties of candy distributed across bowls). We found no clear evidence of partition dependent choice in children (Experiment 1) and no evidence at all of partition dependence in adults' choices (Experiments 1-3). This was true even when methods were closely matched to those of Fox et al.'s Study 4 (Experiment 3). We conclude that the candy-bowl choice task does not reliably elicit partition dependence and propose possible explanations for the discrepancy between these findings and prior reports. Future work
\end{abstract}

Author note We thank the participating families; the Connecticut Science Center; research assistants Jessica Taggart, Ellen Lesser, Jillian Roberts, Rachel Warner, Madeline Kidd, Ziyue Li, Portia Lundie, Anna Schwab, Zoe Feingold, Sarah Seo, Pinzhen Lyu, Sonia Zavala, Ania Rojek, and Suet Ning Wong. We also thank Rebecca Ratner and Craig Fox for helpful input. This work benefited from a Stipend Award from the National Living Laboratory Initiative with funding from NSF under Award Number 1113648, a Wesleyan University Project Grant, NSF DRL-0950252, and NSF DRL-1561214.

Hilary C. Barth

hbarth@wesleyan.edu

1 Department of Psychology, Wesleyan University, 207 High St, Middletown, CT 06459, USA

2 Rutgers University-Newark, New Brunswick, NJ, USA will explore the conditions under which partition dependence in consumer choice does reliably arise.

Keywords Partition dependence $\cdot$ Diversification bias . Decision making

When people choose multiple options simultaneously from a set of possibilities, they tend to make diverse selections rather than choosing multiple instances of the same option, even if one option is preferred. This is referred to as a diversification bias (Read, Antonides, van den Ouden, \& Trienekens, 2001; Simonson, 1990; see also Simonson \& Winer, 1992). Because diversification can occur not only over individual options but also over groups of options, diversification can lead to a phenomenon called partition dependence: the subjective or arbitrary grouping of the options can influence decisions (Fox \& Rottenstreich, 2003). For example, imagine two adults each buying snacks for children's sports teams, looking at the same case of frozen treats containing cherry ice pops, strawberry ice-cream sandwiches, and vanilla ice-cream bars. These people might diversify across different categories, leading to different purchasing patterns (Fox, Bardolet, \& Lieb, 2005). One might subjectively partition the treats into ice-cream versus non-ice-cream groupings, in which case approximately half the selections would consist of cherry ice pops and the other half would consist of strawberry ice-cream sandwiches and vanilla ice-cream bars. The other person might subjectively partition the treats into fruit versus nonfruit groupings; then, half the selections would consist of cherry ice pops and strawberry ice-cream sandwiches and the other half of vanilla icecream bars. This kind of differential grouping, which can be subjectively generated by a decision maker or incorporated into a decision situation by choice architects, biases people away from some selections and toward others. 
Partition dependence in choice has been observed experimentally in several studies. For example, when participants made multiple candy selections from three bowls containing a total of four types of candy, they appeared to diversify their choices across bowls as well as across types of candy (Fox, Ratner, \& Lieb, 2005). In another study, business students' selections of snack preferences from descriptions of familiar snacks were influenced by whether the menu grouped options as "cookies", "crackers", and "fruits and vegetables" versus "cookies and crackers", "fruits", and "vegetables" (Fox et al., 2005). Diversification bias and partition dependence also arise in resource allocation decisions in which resources such as time or money must be allocated to different options. Employees have been found to diversify as they distribute retirement resources, allocating them evenly across options in 401(k) plans (Benartzi \& Thaler, 2001). Experimentally manipulating the partitioning of available options changes people's patterns of diversification: when students allocated free lunches to be claimed over different portions of an academic year, subdividing the first semester (but not the second) into quarters led to more lunches being allocated to the first semester, while the reverse was the case when only the second semester was subdivided into quarters (Fox et al., 2005). The influence of partitioning on decisions is a broad phenomenon, demonstrated in a wide range of contexts (e.g. Fox \& Rottenstreich, 2003; Langer \& Fox, 2005; See, Fox, \& Rottenstreich, 2006; Sonnemann, Camerer, Fox, \& Langer, 2013; Tannenbaum et al., 2015). Because of its broad applicability, partitioning has been proposed as a technique for nudging people to make better decisions (Johnson et al., 2012; Thaler \& Sunstein, 2008).

The present work aims to replicate and extend prior findings of partition dependence in the candy-bowl choice task mentioned above. We chose this simple task to replicate in adults and to extend to younger age groups, in order to ask whether children also show evidence of partition dependence in a choice task. The first evidence of partition dependence in children in a resource allocation task was recently reported by Reichelson and colleagues (Reichelson, Zax, Patalano, \& Barth, 2017). Children (3 to 11 years of age) were influenced by the physical partitioning of hypothetical zoo animals in their allocation of food tokens to the animals. However, no work has yet considered partition dependence in children in a choice task. The candy-bowl choice task is unusual in the partition dependence choice literature in that it is concrete, relies on perceptual rather than conceptual partitioning of options, and has an immediate and certain outcome. The task is thus important in its own right, as an example of a common type of consumer decision, and it is valuable as a paradigm that can be readily extended to other populations.

In the candy-bowl choice task, participants must choose from a physically partitioned menu of items: They are asked to choose five pieces of candy from three bowls containing a total of four types of candy divided over three bowls. Two bowls each contain one type, and a third bowl contains the remaining two types, each placed in one half of the bowl. Thus, the bowls create a three-way partition, but candy type creates a four-way partition. If people distribute choices solely over candy type (with no influence of the candy's arrangement across the bowls), half of their selections should be drawn from the two single-candy bowls combined and half should be drawn from the double-candy bowl. If, however, people distribute choices only across bowls (and candy type plays no role), two thirds of candies should be drawn from the single-candy bowls combined, and one third should be drawn from the double-candy bowl. In Fox, Ratner, (2005), participants' averages fell between these extremes, illustrating that choice appeared to be driven by both candy type and bowl partition. This partition dependence effect has generally been interpreted as arising through a diversification mechanism, with people spreading choices evenly across the available groupings and both candy grouping and bowl grouping playing a role (Fox et al., 2005; Fox et al., 2005).

\section{Experiment 1}

We asked whether participants' choices from a menu of simultaneously presented candy options would be influenced by the arbitrary physical partitioning of the options. The present candy-bowl task used the methods of Fox et al. (2005), except as noted otherwise.

\section{Method}

Participants Seventy adults $\left(M_{\text {age }}=20.4\right.$ years, range $=18$ 22 years, 35 females $)$ and 80 children $\left(M_{\text {age }}=6 ; 10\right.$, range $=$ 4;0 - 9;7, 40 females) participated and were included in analyses. Eleven additional children were excluded due to noncompliance or interference from guardians.

Procedure Adults completed the task at a university student center or in a laboratory room. Children participated during candy-themed holiday events at a science center and a local school. Four types of candy were distributed across three bowls. Because the candy types in the original study of Fox et al. (2005) were unsuitable for young children (e.g., mints, potential choking hazards), small chocolate bars were used here instead (Twix, Hershey's, Kit Kat, and Nestlé Crunch). There were two possible physical arrangements of the candy types and two possible bowl orders, for a total of four counterbalancing conditions. The two possible physical arrangements of the candy types were as follows: Types 1 and 2 appeared in single-candy bowls and Types 3 and 4 appeared in the double-candy bowl, or Types 3 and 4 appeared in the single-candy bowls and Types 1 and 2 appeared in the double- 
candy bowl. Two bowl orders were used to counterbalance left-to-right bowl positions. ${ }^{1}$ The orders were, from left to right, single/single/double and double/single/single. This counterbalancing ensures that participants who simply select five candies in sequential order from left to right do not systematically influence the tally of choices from each bowl type. There was one other procedural difference from Fox et al.'s task in addition to the change in candy types: We used clear bowls (the original study used bowls of three different colors, though bowl color was not described in the published report). Each candy type appeared an equal number of times in each type of bowl in each position. Unopened full bags of each candy type were displayed behind the bowls to illustrate that no candy was scarce.

Participants were asked to choose five candies to keep from the three bowls in front of them. Choices were not visible to those waiting to participate. We recorded the amount of candy chosen of each type from each bowl and the order in which each piece of candy was chosen. After the choice task, participants were asked which one of the candy types they preferred.

\section{Results and discussion}

Adults did not choose more than half (2.5) of the candies from the single-candy bowls $(M=2.50, S D=1.10)$ as would be predicted if the bowl partitions influenced their choices, $t(69)$ $=0, p=.500$, one-tailed. There were no effects of bowl order on choice (average number chosen from the single-candy bowl did not differ across conditions, $p>.250$ ). The data revealed no evidence of partition dependence: The arbitrary distribution of the candies across bowls did not affect adults' choices of candy.

Children chose marginally more than half their candies overall from the two single-candy bowls $(M=2.70, S D=$ $1.10), t(79)=1.63, p=.053$, one-tailed. Although these findings initially appeared consistent with partition dependence, further inspection revealed that the difference arose from the extreme choices, or "antidiversification", of a small number of individuals. When data from participants who chose all five of the same candy type were excluded ( $n=5$ children), the remaining children did not choose more than half from the single-candy bowls $(M=2.55, S D=0.95), t(74)=0.43, p=$ .340 , one-tailed. There were no effects of bowl order on choice (average number chosen from the single-candy bowl did not differ across conditions, $p>.250$ ). Thus, there is no evidence of diversification-based partition dependence in the group-level choice behavior of the remaining children (75/80 participants).

\footnotetext{
${ }^{1}$ Counterbalancing of left-to-right bowl positions across participants was not specified in the previous research report but was included in the design (C. R. Fox, personal communication, May 2017).
}

Given the child results, we asked whether antidiversifiers could have influenced adults' group-level results. We reanalyzed the adult data after removing participants who chose all five of the same candy type $(n=4)$, but results did not change; the choices from the single-candy bowls $(M=$ $2.50, S D=0.95)$ again did not differ from 2.5 candies, $t(65)$ $=0, p=.500$, one-tailed.

This experiment found no evidence of partition dependence in the candy-bowl task in adults. Children also did not appear to make partition-dependent choices. Though we did observe in children the overall group-level pattern of choice that is sometimes interpreted as evidence of partition dependence, here it was driven entirely by five children who chose five of the same type of candy. Thus, the mechanism that is usually implicated as the source of partition dependence in choice tasks of this kind, a bias toward diversification, did not lead to partition dependence in this task. We return to this issue in the general discussion.

\section{Experiment 2}

The adult findings from Experiment 1 are particularly unexpected given prior work reporting that adults' choices were indeed influenced by the way candies were placed in bowls (Fox et al., 2005), and the child findings are ambiguous at best. Before pursuing further studies with children, we aimed to determine whether the original finding could be replicated with adults. We therefore repeated the same study with a new group of adults.

\section{Method}

Participants Sixty-nine adults $\left(M_{\text {age }}=20.7\right.$ years, range $=$ 20-25 years, 45 females) participated.

Procedure The procedure was identical to that of Experiment 1.

\section{Results and discussion}

Adults did not choose more than half their candies from the single-candy bowls $(M=2.59, S D=1.13), t(68)=0.69, p=$ .245 , one-tailed. As in Experiment 1, we reanalyzed the data after removing participants who chose all five of the same candy type $(n=7)$; the choices from the single-candy bowls $(M=2.48, S D=0.84)$ again did not differ from 2.5 candies, $t(61)=-0.15, p=.441$, one-tailed. There were no effects of bowl order on choice (average number chosen from the single-candy bowl did not differ across conditions, $p>$ .250). There was no evidence of partition dependence in this task; the arbitrary distribution of the candies across bowls did not affect adults' choices of candy. 
Experiment 2, like Experiment 1, found no evidence of partition dependence in the candy-bowl task in adults. However, because these experiments did not use a procedure identical to that of Fox et al. (2005), we cannot rule out the possibility that slight procedural differences led to the present failure to find partition dependence effects. Experiment 3 tests this possibility.

\section{Experiment 3}

Experiments 1 and 2 found no evidence of partition dependence in the candy choice task in adults, and no clear evidence in children. Because prior work (Fox et al., 2005) reported that the same task did produce partition dependence in adults, this result is unexpected. However, it is possible that slight procedural differences were responsible for the difference in results. Experiment 3 tests this possibility. To our knowledge, the only such procedural differences in the choice task were the specific candy types and bowl colors used. In Experiment 3, we again used the procedure of Experiments 1 and 2, except that we used three different colored bowls as in Fox et al. (2005), and we used the same candy types. If these are critical dimensions, we should observe partition dependence in this study.

\section{Method}

Participants Eighty adults $\left(M_{\mathrm{age}}=20.6\right.$ years, range $=18-42$ years, 46 females) participated.

Procedure The method was identical to that of the previous experiments, except that we used the same candy types (Smarties, Bazooka Bubble Gum, Tootsie Rolls, and Starlight Mints) as Fox et al. (2005), and used distinctly colored bowls (yellow, green, and orange). Adult participants were again tested in a laboratory setting or student center. Counterbalancing was as described previously.

\section{Results and discussion}

Participants again did not choose more than half the candies from the single-candy bowls $(M=2.49, S D=1.52), t(79)=$ $0.07, p=.471$, one-tailed. Results were similar when we excluded participants $(n=11)$ who chose all five of the same candy type from the single-candy bowls $(M=2.52, S D=$ $1.29), t(68)=0.14, p=.444$, one-tailed. An independentsamples $t$ test revealed an effect of bowl order on choice in the full sample; fewer candies were chosen from single-candy bowls in the single/single/double condition $(M=2.00, S D=$ 1.32) than the double/single/single condition $(M=2.98, S D=$ 1.56), $t(78)=-3.02, p=.003$, two-tailed. The bowl order effect was not significant without the 11 participants who chose all five of the same candy type $(M=2.29, S D=1.15$ vs. $M=2.76$,
$S D=1.39$, respectively, for two bowl orders), $t(67)=-1.56, p$ $=.120$, two-tailed (suggesting that the effect may have been driven largely by the chance locations of the candy types chosen by individuals with particularly strong preferences). As in Experiments 1 and 2, there was no evidence of partition dependence in this task; the arbitrary distribution of the candies across bowls did not affect choices.

\section{General discussion}

In three experiments, we investigated the phenomenon of partition dependence in children's and adults' consumer choice, specifically in the candy-bowl choice task of Fox et al. (2005). The goal was to replicate prior findings of partition dependence in a simple choice task in adults, and to extend the task to younger populations. In children, partition dependence has previously only been demonstrated in a resource allocation task involving allocation of food tokens to physically partitioned zoo animals (Reichelson et al., 2017). The present experiments yielded no evidence that partitioning shaped choice in adults in the candy-bowl task and yielded no clear evidence of partition dependence in children in this choice task.

Experiments 1 and 2 used the task of Fox et al. (2005) with different candy types and bowl colors. In both studies, adults did not choose more candies from the single-candy bowls than from the double-candy bowls (the predicted result if the partitioning of the candy into bowls influenced selections). Experiment 3's procedure was closely matched to that of previous work, using the same candy types and bowl colors as Fox et al. (2005), but the same results emerged: again there was no evidence of partition dependence.

Our adult findings clearly differ from previous findings of partition dependence in adults' choice with the candy-bowl task (Fox et al., 2005). We suggest three potential explanations. First, previous work shows that strong preference, like expertise in a domain, reduces partition dependence (e.g. Fox et al., 2005). It could be that the participants tested in the prior study ( $N=74$ undergraduate students) had weaker candy preferences and were therefore more susceptible to the influence of the bowl partition than were our participants. Although there is no a priori reason to predict such a difference, this is a possibility. Second, although procedures were very similar across studies, their settings differed in ways that might matter. In the prior study, students received course credit for research participation and completed the candy choice as part of an hour-long set of tasks. In our study, the task was not embedded in an experimental context; nearly all of the adults chose candy during a brief encounter in the student center, without going through written consent due to the minimal nature of the task. Similarly, all of the children chose candy in an entirely naturalistic setting. One could speculate that if participant expectancy effects or participant fatigue influence this task, this 
alteration could account for the present nonreplications. A third possible explanation for the difference is simply that the appearance of partition dependence in the previous report might have been due to chance. We cannot rule out any of these explanations, but all point to the conclusion that the candy-bowl task does not robustly elicit partition dependence in adults.

In our view, the child data from Experiment 1 should also not be interpreted as evidence of partition dependence in children's choice. In Experiment 1, children's candy choices initially appeared consistent with partition dependence (more candies taken from single-candy bowls overall), but the effect was entirely driven by a handful of individuals $(N=5$ out of 80) who chose all five candies of the same type, all of which happened to be located in single-candy bowls. Without these antidiversifiers, there was no evidence of partition dependence in the group. Although candy locations were varied so that, on average, people's preferred candies would ideally be distributed evenly across bowl types, in practice the highly specialized choices of a few individuals can fall in single-item bowls (or not) and can create an illusion of group-level partition dependence effects. In other words, apparent (but spurious) partition effects might be present or absent depending on the chance locations of preferred items. ${ }^{2}$

We believe the chance locations of a few children's highly specialized choices in fact created the spurious appearance of partition dependence in the overall group in Experiment 1, which disappeared when we analyzed data from the remaining $94 \%$ of the children. This interpretation of the child data, taken together with the absence of any evidence for partition dependence in three adult studies, suggests that there is no reliable evidence of partition dependence in the candy-bowl choice task. This conclusion is consistent with prior work showing that increased cognitive load in the adult candy choice task, which might be predicted to increase partition dependence effects as has occurred in other studies, instead attenuated them (Fox et al., 2005). ${ }^{3}$

It could be claimed, conversely, that these child results do provide evidence of partition-dependent consumer choice after all. Partition dependence in multiple-item choice, as in the candy choice task, is usually interpreted as being a product of diversification, and clearly diversification cannot have produced partition dependence in the current data. However, while diversification is one strategy that can lead to partition dependence, it has been proposed that other psychological

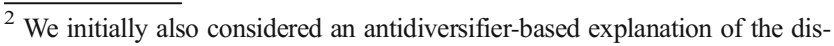
crepancy between the adult findings here and those of Fox, Ratner, \& Lieb (2005). However, findings of a reanalysis of the Fox et al. data refuted this explanation: The partition dependence effect remained even when all antidiversifiers (15\% of participants) were excluded from analysis (C. R. Fox, personal communication, May 2017).

${ }^{3}$ For details of a different explanation - that the increased load might reduce partition dependence under some circumstances while increasing it in otherssee Fox, Bardolet, and Lieb (2005).
}

mechanisms must also play a role - in part because the effect arises in single-item choice, when participants have no opportunity to diversify (Tannenbaum, et al., 2015; Tannenbaum, Fox, \& Goldstein, n.d.). If such an alternative mechanism is in fact involved in the candy choice task, our findings could provide valid evidence of partition dependence in children: From that perspective, the effect observed here simply comes from some mechanism other than diversification that was only exhibited within the five children who antidiversified, and it comes only from mechanism(s) other than diversification (because the diversifying children showed no partition dependence).

Partition dependence is a broad phenomenon with strong empirical support; it has been demonstrated in lab studies and observed in field situations in many different contexts in addition to consumer choice, such as resource allocation. The present work challenges the evidence for partition dependence specifically using the candy-bowl task. Data from this task have been used to support the claim that arbitrary perceptual partitions influence choices of multiple options from an array. To our knowledge, this is the only task used to provide evidence for partition dependence under these conditions. The candy-bowl task differs from other choice tasks in which partition dependence has been found in adults (tasks involving wine selection and snack selection; Fox et al., 2005) in several ways. Unlike other tasks, the candy-bowl task involves perceptual groupings that are arbitrary rather than conceptually coherent or meaningful, and few choice options within each grouping (thus, the groupings do little to simplify the task). The present studies leave open the question of which critical qualities of a decision situation do lead to robust findings of partition dependent behavior. A first step for future work is to identify the conditions under which partition dependence in consumer choice does reliably arise.

\section{References}

Benartzi, S., \& Thaler, R. H. (2001). Naive diversification strategies in defined contribution saving plans. The American Economic Review, 91, 79-98.

Fox, C. R., Bardolet, D., \& Lieb, D. S. (2005). Partition dependence in decision analysis, resource allocation, and consumer choice. In R. Zwick \& A. Rapoport (Eds.), Experimental business research (Vol. 3, pp. 338-360). Dordrecht, The Netherlands: Kluwer.

Fox, C. R., Ratner, R. K., \& Lieb, D. S. (2005). How subjective grouping of options influences choice and allocation: Diversification bias and the phenomenon of partition dependence. Journal of Experimental Psychology: General, 134, 538-551.

Fox, C. R., \& Rottenstreich, Y. S. (2003). Partition priming in judgment under uncertainty. Psychological Science, 14, 195-200.

Johnson, E. J., Shu, S. B., Dellaert, B. G. C., Fox, C., Goldstein, D. G., Haübl, G., \& Weber, E. U. (2012). Beyond nudges: Tools of a choice architecture. Marketing Letters, 23, 487-504. 
Langer, T., \& Fox, C. R. (2005). Nä̈ve diversification and partition dependence in investment allocation decisions: An experimental investigation. Unpublished manuscript.

Read, D., Antonides, G., van den Ouden, L., \& Trienekens, H. (2001). Which is better: Simultaneous or sequential choice? Organizational Behavior and Human Decision Processes, 84, 54-70.

Reichelson, S., Zax, A., Patalano, A. L., \& Barth, H. (2017). Partition dependence in development: Are children's decisions shaped by the arbitrary grouping of options? Manuscript submitted for publication.

See, K. E., Fox, C. R., \& Rottenstreich, Y. S. (2006). Between ignorance and truth: Partition dependence and learning in judgment under uncertainty. Journal of Experimental Psychology: Learning, Memory, and Cognition, 32, 1385-1402.

Simonson, I. (1990). The effect of purchase quantity and timing on variety seeking behavior. Journal of Marketing Research, 32, 150-162.
Simonson, I., \& Winer, R. S. (1992). The influence of purchase quantity and display format on consumer preference for variety. Journal of Consumer Research, 19, 133-138.

Sonnemann, U., Camerer, C. F., Fox, C. R., \& Langer, T. (2013). How psychological framing affects economic market prices in the lab and field. Proceedings of the National Academy of Sciences, 110, 11779-11784.

Tannenbaum, D., Doctor, J. N., Persell, S. D., Friedberg, M. W., Meeker, D., Friesema, E. M.,. . . Fox, C. R. (2015). Nudging physician prescription decisions by partitioning the order set: Results of a vignette-based study. Journal of General Internal Medicine, 30, 298-304.

Tannenbaum, D., Fox, C. R., \& Goldstein, N. J. (n.d.). Partitioning menu items to nudge single-item choice (Working paper). Retrieved from https://davetannenbaum.github.io/documents/pdepend.pdf

Thaler, R., \& Sunstein, C. (2008). Nudge: Improving decisions about health, wealth, and happiness. New Haven, CT: Yale University Press. 